\title{
MINIMUM COST TOPOLOGICAL ORDERING
}

\author{
THINH D. NGUYEN
}

\begin{abstract}
We are given an $n$ vertex directed graph $G=(V, E)$ and also given a cost function $c: V \times[n] \rightarrow \mathbb{R}$. Consider a topological ordering of the vertices, $v_{1}, \ldots, v_{n}$, the cost of the ordering is $\sum_{i=1}^{n} c\left(v_{i}, i\right)$. We shall prove that finding the minimum cost topological ordering is NP-hard.
\end{abstract}

\section{Proof of hardness}

We show a reduction from the Shuffle Problem: given words $w, w_{1}, \ldots, w_{k}$ over the alphabet $\{a, b, c\}$, decide whether $w$ can be obtained as an interleaving (aka "shuffle") of $w_{1}, \ldots, w_{n}$. This problem is NP-hard as shown by Warmuth \& Haussler [3, Theorem 3.1].

Given an instance $w, w_{1}, \ldots, w_{n}$ of this problem, and writing $l_{i}:=\left|w_{i}\right|$ for all $1 \leq i \leq n$, we build the DAG $G$ as a union of path graphs $L_{1}, \ldots, L_{n}$, where each $L_{i}$ for $1 \leq i \leq n$ has $l_{i}$ vertices written $v_{1}^{i}, \ldots, v_{l_{i}}^{i}$. Now, we define the cost function $f$ as follows: for each $1 \leq i \leq n$ and $1 \leq j \leq l_{i}$, for each $1 \leq k \leq|w|$, we set $f\left(v_{j}^{i}, k\right)$ to be 0 if the $j$-th character of $w_{i}$ is the same as the $k$-th character of $w$, and 1 otherwise.

This reduction is clearly in PTIME, and it is clear that the minimum cost of a topological sort is 0 iff there is an interleaving of the path graphs realizing exactly the word $w$, showing that the reduction is correct.

\section{Conclusion}

Garey and Johnson [1] shapes their theory based on previous primal works of Cook, Levin and Karp. Johnson [2] moves on with the guide to this theory. As long as we study a mathematical conjecture, we should encourage ourselves of having enough labouring hours on popular maths books like these. Then, reading some articles on theory of computing like [4] is a good practice. Only after that, could we think of the ultimate final for all mathematics sciences.

\section{REFERENCES}

1. Michael R. Garey, David S. Johnson, Computers and Intractability: A Guide to the Theory of NP-Completeness

2. David S. Johnson, The NP-Completeness Column: An Ongoing Guide. pp.393-405

3. Manfred K. Warmuth, David Haussler, On the complexity of iterated shuffle, Journal of Computer and System Sciences 28, pp.345-358 (1984)

4. Phan Dinh Dieu, Le Cong Thanh, Le Tuan Hoa, Average Polyno-mial Time Complexity of Some NP-Complete Problems, Theor. Comput. Sci. 46(3): pp.219-237 (1986)

Current address: Department of Mathematics, Moscow State University

Email address: kosmofarmer@yandex.com

Key words and phrases. graph, order, sort, topological.

Perebor. 